\title{
Administrative Structures for Education Programs
}

\section{INTRODUCTION}

Discussions about the way to organize and manage an education program within a library usually result in three options: a librarian within the reference department is assigned responsibility for the program, a coordinator is appointed with authority to enlist the help of librarians throughout the organization, or a separate department is established to run the program. Two years ago, the staff of Information Management Education Services (IME) in the Health Sciences Library at the University of North Carolina at Chapel Hill discussed other options as part of a strategic planning effort underway at the library. Nine possible models came out of a series of brainstorming sessions. These are outlined below with the advantages and disadvantages of each structure as viewed from the IME department setting. Major considerations underlying these models were the level of visibility that the model would provide to education, whether staff would be shared with other departments, and where leadership for educational programs would reside. The models are not listed in any particular order.

\section{POSSIBLE MODELS}

A separate department with a department head reporting to the library director was the model in place at the Health Sciences Library at the time of the discussions described above. The following were viewed to be the advantages and disadvantages of this model.

Advantages:

- demonstrates commitment to education;

- gives internal status to the program;

- enables high visibility; national as well as local and regional presence were achieved rapidly with this model;

- $\quad$ provides a dedicated advocate (department head) for education programs;

- guarantees participation/input into major library-wide decisions;

- $\quad$ provides better communication between IME staff and rest of the library staff and administration;

- provides a resource person (department head) who is actually involved in the programs;

- removes competition for the department head's time;

- enables staff to develop and improve teaching skills;

- enables higher level of quality control of support materials;

- provides continuity with schools and departments outside the library;

- enables offering a higher volume and greater variety of programs; -enhances continuity within the IME programs;

- ensures the teaching staff are genuinely interested in education; 
- improves ability to adapt to the rhythm of the school year;

- removes conflict for time between project planning and on-demand services, thus keeping the priority for education high.

Disadvantages:

- increases the number of department heads;

- makes recruiting more difficult because positions are specialized;

- makes cross-training with reference and computing services necessary;

- costs more to have a department head and staff with special expertise, equipment, and space;

- can only be implemented in larger libraries;

- may reduce flexibility in scheduling because staff have specialized roles.

A second model would be the same as the first except that a coordinator would head the unit and report to a department head.

Advantages:

- same as the first model as they pertain to a staff dedicated to education;

- costs less for a coordinator than a department head;

- potential is better for cross-over knowledge being shared with the department in which the education unit is located.

Disadvantages:

- potential exists for conflicts of interest on the part of the department head;

- dilutes the education perspective;

- conveys less status, visibility, and commitment to education;

- coordinator lacks direct participation in the management team;

- department head may not be agreeable to or understand the education planning mode.

A third model follows the lines of the two above except that there is no coordinator; the education unit reports directly to the department head who serves as coordinator.

Advantages:

- same as the first model as they pertain to a staff dedicated to education;

- costs even less than the first two models.

Disadvantages:

- same as in the second model, only exaggerated;

- the department head has a heavier workload and more competition for resources;

- lack of a dedicated coordinator may adversely affect recruiting. 
A fourth model would combine education completely with an existing department; responsibility for education would be shared.

Advantages:

- personnel costs are less than for the previously discussed models;

- cross-over knowledge is potentially good;

- if combined with reference, exposure to certain types of user questions is greater, facilitating instruction pertaining to those areas.

Disadvantages:

- the flip side of the first model's advantages of having a dedicated staff, e.g., lack of expertise/interest on the part of some instructors;

- could result in a decrease in the type of programs offered;

- staff must deal with conflicting tasks;

- same disadvantages as the second and third models;

- department head assumes all responsibility for administration, expertise, and resources;

- removes a career ladder step, i.e., from reference to specialization in education.

A fifth model would combine education with another department while maintaining a coordinator but not a dedicated staff. This is one of the most common models in place at the present time.

Advantages:

- some administrative tasks are assumed by the coordinator;

- personnel costs are less than those models with a dedicated staff;

- other advantages are the same as for the fourth model.

Disadvantages:

- the coordinator must recruit and train teachers while dealing with the disadvantages of model four, particularly a staff with other tasks demanding attention;

- leaves little time for planning and preparation of teaching material.

A sixth model is to have a department head for education who has the responsibility and authority to contract with other departments in the library for projects and hours.

Advantages:

- works better for workshops and orientations;

- helps in obtaining good subject expertise of instructors;

- easily allows scaling back on education programs.

Disadvantages: 
- difficult to use this model with course-related or credit courses in which continuity and duplication are issues;

- poses a staff development problem if instructors lack teaching skills;

- it is difficult to obtain and maintain instructors' commitment to non-primary functions;

- increases the number of department heads with a one-person department;

- increases the difficulty of recruiting for the department head position;

- increases the difficulty of starting new programs;

- may necessitate hiring more staff in other departments.

A seventh model is to only provide one-on-one instruction, i.e., to have no education department or programs.

Advantages:

- costs little if there is no commitment to build up the reference staff;

- user receives instruction at the time of expressed need, depending on the reference staff's availability.

Disadvantages:

- docs not meet significant demand for group instruction;

- the library may not have the option of referring requests for group instruction to other libraries;

- not cost-effective on the part of the reference staff, i.e., much effort is duplicated;

- counter to trends in medical librarianship.

An eighth model consists of centralizing leadership for education programs in the library but recruiting instructors from outside the library.

Advantages:

- personnel costs are kept inexpensive;

- strengthens potential of having instructors with subject expertise;

- potentially broadens the visibility for the program;

- increases credibility of instructors with the audience as content pertains to their discipline;

- builds a pool of more "educated" users who could serve as library advocates on other occasions.

Disadvantages:

- introduces lack of control/autonomy of the library's programs;

- instructors may lack commitment to the library; the rate of turnover of instructors may increase;

- difficult to recruit for instructors because of their time constraints and the lower rewards for teaching at many institutions;

- introduces the possibility of lack of teaching expertise, knowledge, and understanding of the library;

- makes educational follow-up more difficult; 
- increases difficulties of communicating with instructors;

- increases difficulty of recruiting an education coordinator;

- may complicate support issues, e.g., materials development, printing, use of equipment and space;

- $\quad$ introduces "training the trainers" issues, e.g., dilution of library knowledge, scheduling, back-up.

The ninth, and final, model consists of elevating education to an "umbrella" department, encompassing such functions as reference, online services, consultation services, and microcomputer services.

Advantages:

- implies the strongest emphasis on education of the models described here;

- increases likelihood that other library services will be viewed from an educational perspective;

- has the same advantages as merging education with other departments.

Disadvantages:

- has the same disadvantages of merging education with other departments;

- may be too large to manage as one department;

- microcomputer user support or other functions may be too different to combine with education or reference.

\section{CONCLUSION}

The above is, by no means, an exhaustive list of models or of advantages and disadvantages. Those who have actually used one or more of these models will see added benefits and drawbacks of them. In addition, some of these pluses and minuses need to be examined from a different library's perspective. However, it is hoped that these may spark some new thoughts on arrangements for providing educational services. If you have comments to share, please feel free to send them to the column editor. Francesca Allegri

806 S. Foley Street

Champaign, IL 61820

Comments and suggestions should be sent to the Column Editor: Francesca Allegri. 